\title{
Location-based Orientation Context Dependent Recommender System for Users
}

\author{
Mr. C. Vijesh Joe,
}

Assistant Professor,

Department of Computer Science and Engineering,

VV College of Engineering,

Tirunelveli, India.

vijesh.joe@gmail.com

Dr. Jennifer S. Raj,

Department of ECE,

Gnanamani College of Technology,

Namakkal, India.

jennifer.raj@gmail.com

Abstract: As the technology revolving around IoT sensors develops in a rapid manner, the subsequent social networks that are essential for the growth of the system will be utilized as a means to filter the objects that are preferred by the consumers. The ultimate purpose of the system is to give the customers personalized recommendations based on their preference. Similarly, the location and orientation will also play a crucial role in identifying the preference of the customer is a more efficient manner. Almost all social networks make use of location information to provide better services to the users based on the research performed. Hence there is a need for developing a recommender system that is dependent on location. In this paper, we have incorporated a recommender system that makes use of recommender algorithm that is personalized to take into consideration the context of the user. The preference of the user is analysed with the help of IoT smart devices like the smart watches, Google home, smart phones, ipads etc. The user preferences are obtained from these devices and will enable the recommender system to gauge the best resources. The results based on evaluation are compared with that of the content-based recommender algorithm and collaborative filtering to enable the recommendation engine's power.

Keywords: IoT devices; orientation context; context awareness; social networks; recommender system 
Journal of trends in Computer Science and Smart technology (TCSST) (2021)

Vol.03/ No. 01

Pages: 14-23

https://www.irojournals.com/tcsst/

DOI: https://doi.org/10.36548/jtcsst.2021.1.002

\section{Introduction}

The quick advancement in social networks and sensing technologies has resulted in an increased data generation on a daily basis. To prevent the information overload, the recommender systems will act as a filtering service. The major activity of this system is to recommend the best suitable objects to the users based on their preferences. A number of challenges have been tackled over the years to build an efficient recommender system (RS) and with each evolving RS, the algorithm is also modified to suit the trending technological advancement. When coping with the RS, there are two aspects that need to be addressed [1]. First is the context in which the system is performed such as preferences, user activity, orientation and location and the next is the recognition of information that is detected. The information that is obtained from social networks will provide a platform for SN detection based on different contextual information. A number of such information have been identified for various RSs that have been formulated to ensure that the user gets a more personalized analysis and experience. User locations, times, devices, social circles, physical environments, interests and activities are some of the key aspects that are taken into consideration in order to build an overall list of contextual information. This information is fed into to RS to identify the individual preferences of the user. Thought these information gathered will help to create a better RS, the location based recommendation will also play a significant role in orientation context. But, over the years, this aspect has not been properly researched over the years. In recent advances in accelerometer sensors, gyroscope and magnetometer along with smart phones have raised the importance of orientation context [2]. In this proposed work, a user pointing direction (UPD) is used to describe the orientation context that uses smart phones to identify the recommendations. Stampede is an example of how UPD will play a crucial role in RSs. If a person decides to spend time at Stampede, the RS will take into consideration his preferences and will further help the user in identifying orientation aware and personalized location recommendations. Though this will affect the RS provided to the user, orientation context and location play a crucial role in large festival that holds multiple exhibitions. The proposed work investigates on the systematic errors that are caused due to sensors malfunctioning. Moreover, this model will work only in an environment where there is availability of GPS signal, preferably outdoors. Evaluating and testing the system indoors is not considered in this paper and focus is mainly on outdoors [3]. Moreover, in this system, we also require information obtained from various services like 
Journal of trends in Computer Science and Smart technology (TCSST) (2021)

Vol.03/ No. 01

Pages: 14-23

https://www.irojournals.com/tcsst/

DOI: https://doi.org/10.36548/jtcsst.2021.1.002

Facebook, Instagram and Twitter [4]. Here virtual communities are detected using these social media networking services. However, there are certain constraints and limitations involved where the users will be required to sign privacy policy agreements to give RS permission to access [5] their information. This paper is organised such that the next session provides a review of the research related to the proposal at hand. Section 3 describes the proposed work and section 4 shows simulation results and discussions based on the results. Section 5 draws conclusion based on section 4 .

\section{Related Works}

The expanding and drawing demand for computational power in terms of electronic devices such as smart phones and laptops make them the most attractive tools that are capable of storing, manipulating and using data [6]. There are many research work done on the orientation in mobile computing that can be used to enhance the way in which different application function such as directional service discovery, augmented reality display and navigation. The most early introduction the recommendation system is that of orientationaware services known as Azim [7]. In this paper, we have introduced a novel recommender algorithm that combines content-based and collaborative filtering methods that makes use of context information such as orientation and location. Another aspect of this work is to address the cold start problem faced by content-based and collaborative filtering recommender algorithms by updating context information. In [8-9] the authors have proposed a novel technique to update user contextual information. This information could also be used as the solution to cold start issue. This issue is one of the most crucial challenges faced by traditional recommender systems. The cloud storage and internet infrastructure that is required are made available for storing as well as transferring the data that is collected. Though this is not limited, the proposed RS is evaluated and implemented considering it to be a recommendation based on the location. Since it has been designed on a common recommender system, it can be implemented in several other areas like route recommendations [10], with ease. In this paper, the user pointing direction is used to determine the orientation context and hence the results thus obtained will not have an impact on the various modes of transportation, provided the location and UPD is measureable [11]. As we progress in developing the RS, it can also be used in the direction of the moving user 
Journal of trends in Computer Science and Smart technology (TCSST) (2021)

Vol.03/ No. 01

Pages: 14-23

https://www.irojournals.com/tcsst/

DOI: https://doi.org/10.36548/jtcsst.2021.1.002

in a particular area such that it will be used to enhance the direction and location context [12]-[13].

\section{Proposed Work}

The paper introduces a recommender system that is based on location and is orientation-aware, built with various tasks such as recommendation making, ambiguity handling, location detection, orientation detection, profile making and item extracting. When compared to other traditional RS that make use of the geographic point alone to determine the location of an object, this work introduces an RS that uses minimum bounding box for every consideration. The main aspect of this system is to use the smart phone to control the devices in a remote manner. By combining data from the accelerometer sensors and magnetic compass, the data obtained is known as azimuth information as observed in the traditional transmission.

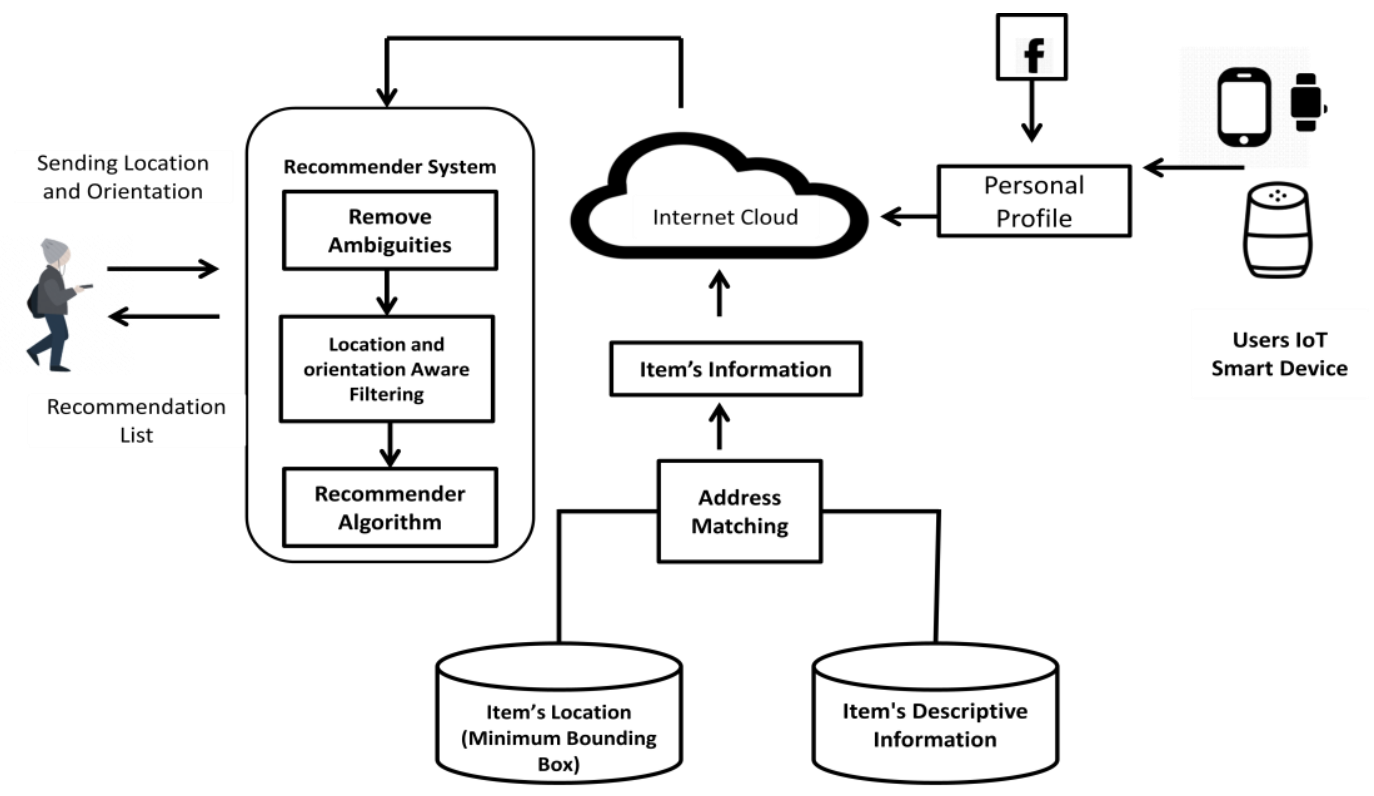

Fig.1. Orientation Aware Mobile Recommender System Architecture

Similarly, human beings' common behaviour is observed as a part of the daily cycle and is used to construct a context-aware recommender system that can be used by the customers on their mobile phones. This mannerism of seeking information is a natural human behaviour and will be used effectively in the recommender system. The travel direction and speed of the user is considered in this proposed work in order to make the recommender 
Journal of trends in Computer Science and Smart technology (TCSST) (2021)

Vol.03/ No. 01

Pages: 14-23

https://www.irojournals.com/tcsst/

DOI: https://doi.org/10.36548/jtcsst.2021.1.002

system more user-friendly. Hence the previous locations as well as the current location of the user are examined to determine the apt direction of user movement. As the first step, the user's pointing direction will be applied and then used in filtering context. The next step would be to determine orientation contexts, location and user preferences with the help of IoT devices used by the users. As the last step, the mobile phones will prove virtual communities from where context information can be obtained to address the cold start problem. A number of studies made and observed so far have deemed the mobile phones to be devices that act as excellent pointing objects. Hence they can be used as a means to interact between the real world objects and human beings by simple signs like pointing at particular objects. The use and implementation of embedded sensors in mobile phones have changed the aspect in which people react and interact with their surroundings. In fact, URQUELL is a successful prototype that was developed based on services that are location-based. Sensor kits worn on the wrists and smart phones were used as devices that piqued user attention through recognition. Similarly, directional information is determined using in-build magnetic field sensors, accelerometers and gyroscope that use integrating sensory data collection.

The idea behind this concept is to decrease the computational cost while enhancing the computational capacity of the system at the same time. It will also be able to represent the object's geographical area in a better manner when compared to the representation of a region using a single geographical location. Information about the object under consideration is extracted from several sources, as seen in Fig.1. A social media service was considered to collect information for social events using location-based recommendation. Similarly, the geographic coordination of the various locations were also stored in a particular database. On using an algorithm to match the addresses on both the database information gathered, the optimal location was identified. Due to the quick growth rate of the number of IoT devices used like the Amazon, Google Home, iPads and other smart devices, there are many opportunities involved in determining the user preferences. In this paper, we have attempted to determine the preferences of a particular user based on the information gathered from the IoT devices that are used by them. However, whenever a person who does not have any information on the social media front joins the recommender system, the cold state problem will arise. This is because the user-matrix developed will draw a blank when it uses the preferences of a user who has no history. Hence the RS will not be able to recommend any location and will not initialise the process. The user profile of a particular person is collected 
Journal of trends in Computer Science and Smart technology (TCSST) (2021)

Vol.03/ No. 01

Pages: 14-23

https://www.irojournals.com/tcsst/

DOI: https://doi.org/10.36548/jtcsst.2021.1.002

by means of the social networks or IoT devices. In this paper, we have created personal profiles from which information is gathered by means of tablets, smart phones, google home etc and is fed into a context reasoning engine.

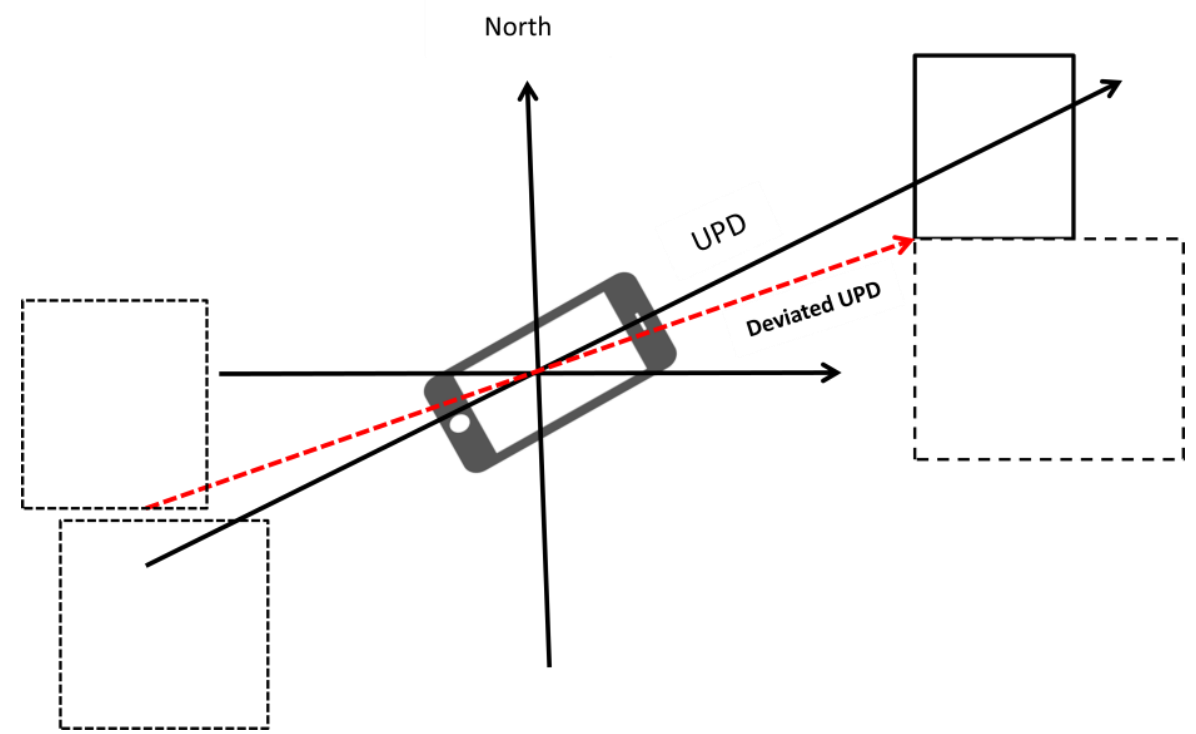

Fig.2. Points allocated along with pointing line with respect to Azimuth reading

When using a device like the smart phone with respect to global coordinate system, as shown in Fig.2, it will provide the azimuth coordinates with the help of orientation sensor. As represented in the figure, we will be using the minimum bounding box in order to determine the objects that are present in the surroundings Moreover the azimuth value measured is used to find the four ordered regions. The bounding box will provide a $\mathrm{y}$ and $\mathrm{x}$-axis dimension within which all the building points should be located. Using the azimuth and smart phone coordinate, the gradient and equation of UPD can be expressed as:

$$
\begin{gathered}
z_{p l}=\tan (\propto) \\
y_{p l}=z_{p l} X_{p l}+y_{u}-z_{p l} x_{u}
\end{gathered}
$$

Where the coordinates are represented as $X_{p l}, Y_{p l}$ and $z_{p l}$ is the gradient. 
Journal of trends in Computer Science and Smart technology (TCSST) (2021)

Vol.03/ No. 01

Pages: 14-23

https://www.irojournals.com/tcsst/

DOI: https://doi.org/10.36548/jtcsst.2021.1.002

\section{Results and Discussions}

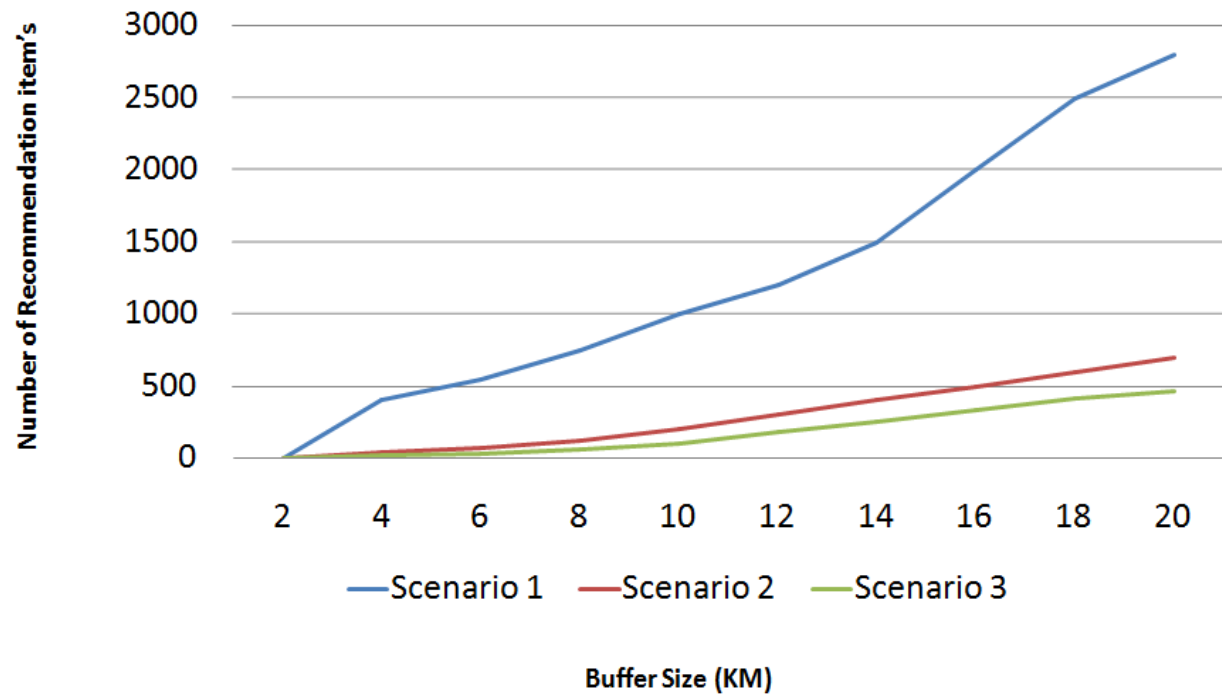

Fig.3 Comparison of proposed RS in 3 scenarios for four buffers without orientation context

A comparison is drawn on the proposed recommender algorithm's performance based on the consideration of 2, 5,10 and $15 \mathrm{Kms}$ as the buffer size without taking into consideration the orientation context as shown in Fig.3. Similarly, Fig.4 shows the comparison based on 4 UPDs of different sizes with a varying difference of $5 \mathrm{Kms}$, starting at $5 \mathrm{Kms}$.

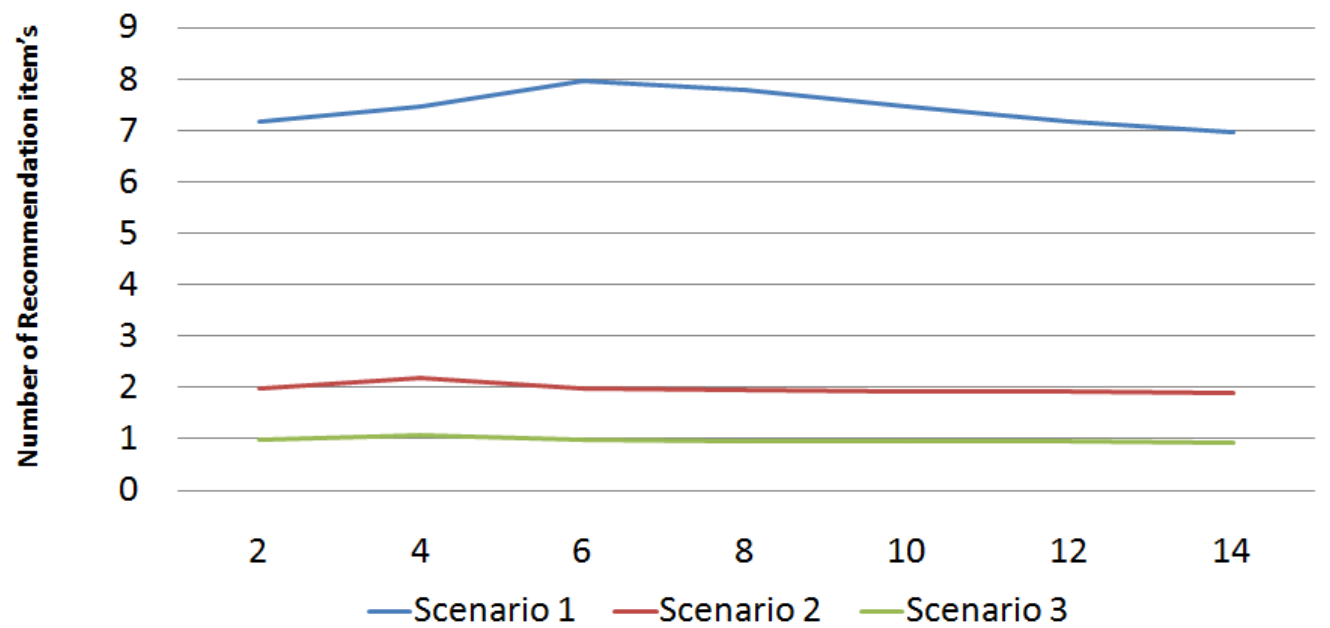

Fig.4 Comparison of proposed RS in 3 scenarios for four buffers using orientation context 
Journal of trends in Computer Science and Smart technology (TCSST) (2021)

Vol.03/ No. 01

Pages: 14-23

https://www.irojournals.com/tcsst/

DOI: https://doi.org/10.36548/jtcsst.2021.1.002

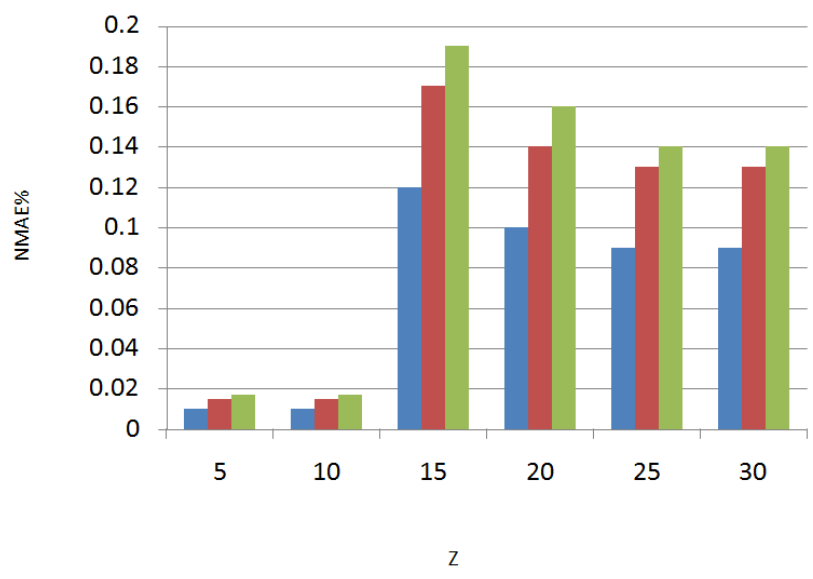

Fig.5 Performance Evaluation using NMAE index

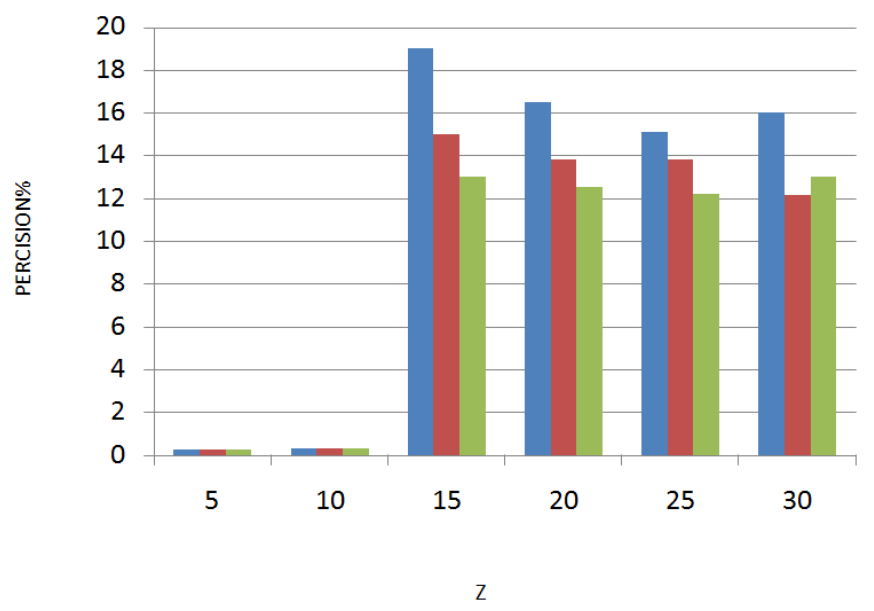

Fig.6 Performance Evaluation using Precision index

Fig.5 and Fig.6 show that the depending on the various recommended events, the value of the index with which they are measured for three recommender algorithms will vary. Fig.5 represents the NMAE index value while Fig.6 represents the precision index value.

\section{Conclusion}

The recommender system is an excellent way to use the data collected from various IoT devices and social networks that are popularly used. Though many attempts have been made in enhancing the recommendations of a use, the aspect of user orientation context was still not used to its full potential. Hence in this paper we have proposed a recommender system that has some advantages over the other traditional systems such as enabling a more 
Journal of trends in Computer Science and Smart technology (TCSST) (2021)

Vol.03/ No. 01

Pages: 14-23

https://www.irojournals.com/tcsst/

DOI: https://doi.org/10.36548/jtcsst.2021.1.002

personalized event recommendation, tackling issues due to errors in sensory data and cold start problems. Here the use of content-based recommender and collaborative filtering is examined in real time using different scenarios and the optimal solution is determined. Based on the role of user orientation, it is identified that the proposed algorithm performs better in terms of personalization level. Future study can be conducted or other location-based recommendations like restaurant recommendations and recreational spots. Similarly other algorithms that have a complexity of generation can also be analysed and compared with the proposed work. Investigation on ways to enhance user privacy can be performed as future work of this paper.

\section{References}

[1] Lu, J., Wu, D., Mao, M., Wang, W., \& Zhang, G. (2015). Recommender system application developments: a survey. Decision Support Systems, 74, 12-32.

[2] Zhang, S., Yao, L., Sun, A., \& Tay, Y. (2019). Deep learning based recommender system: A survey and new perspectives. ACM Computing Surveys (CSUR), 52(1), 1-38.

[3] Joseph, S. I. T., \& Thanakumar, I. (2019). Survey of data mining algorithm's for intelligent computing system. Journal of trends in Computer Science and Smart technology (TCSST), 1(01), 14-24.

[4] Ge, Y., Xiong, H., Tuzhilin, A., Xiao, K., Gruteser, M., \& Pazzani, M. (2010, July). An energy-efficient mobile recommender system. In Proceedings of the 16th ACM SIGKDD international conference on Knowledge discovery and data mining (pp. 899-908).

[5] Levandoski, J. J., Sarwat, M., Eldawy, A., \& Mokbel, M. F. (2012, April). Lars: A location-aware recommender system. In 2012 IEEE 28th international conference on data engineering (pp. 450-461). IEEE.

[6] Gomez-Uribe, C. A., \& Hunt, N. (2015). The netflix recommender system: Algorithms, business value, and innovation. ACM Transactions on Management Information Systems (TMIS), 6(4), 1-19.

[7] Senthilkumar, M., Kavitha, V. R., Kumar, M. S., Raj, P. A. C., \& Shirley, D. R. A. (2021, March). Routing in a Wireless Sensor Network using a Hybrid Algorithm to Improve the Lifetime of the Nodes. In IOP Conference Series: Materials Science and Engineering (Vol. 1084, No. 1, p. 012051). IOP Publishing. 
Journal of trends in Computer Science and Smart technology (TCSST) (2021)

Vol.03/ No. 01

Pages: 14-23

https://www.irojournals.com/tcsst/

DOI: https://doi.org/10.36548/jtcsst.2021.1.002

[8] Sun, Y., \& Zhang, Y. (2018, June). Conversational recommender system. In The 41st international acm sigir conference on research \& development in information retrieval (pp. 235-244).

[9] Manoharan, S. (2020). Patient Diet Recommendation System Using K Clique and Deep learning Classifiers. Journal of Artificial Intelligence, 2(02), 121-130.

[10] van Capelleveen, G., Amrit, C., Yazan, D. M., \& Zijm, H. (2019). The recommender canvas: a model for developing and documenting recommender system design. Expert systems with applications, 129, 97-117.

[11] Tejeda-Lorente, Á., Porcel, C., Peis, E., Sanz, R., \& Herrera-Viedma, E. (2014). A quality based recommender system to disseminate information in a university digital library. Information Sciences, 261, 52-69.

[12] Vijayakumar, T., \& Vinothkanna, R. (2020). Capsule Network on Font Style Classification. Journal of Artificial Intelligence, 2(02), 64-76.

[13] Alhijawi, B., \& Kilani, Y. (2020). The recommender system: A survey. International Journal of Advanced Intelligence Paradigms, 15(3), 229-251. 